\section{Prävalenz von Schlaganfall und transitorischer ischämischer Attacke (TIA) bei obstruktiver Schlaf-Apnoe: eine retrospektive Erhebung an 187 konsekutiven Patienten ${ }^{1}$}

\begin{abstract}
Zusammenfassung: Hintergrund: Die obstruktive Schlaf-Apnoe (OSA) ist vermutlich signifikant mit zerebrovaskulären $\mathrm{Er}$ krankungen assoziiert. Epidemiologische Daten, die einen solchen Zusammenhang sicher belegen, fehlen jedoch noch weitgehend. Deswegen war das Ziel der vorliegenden retrospektiven Untersuchung, Anhaltspunkte für die Prävalenz von Schlaganfall und transitorischer ischämischer Attacke (TIA) bei OSA zu gewinnen. Patienten und Methode: 187 konsekutive Patienten mit einer polysomnographisch gesicherten Diagnose einer OSA, die während des Zeitraums von 1/1998 bis 3/2000 in unserem Schlaflabor untersucht worden waren, wurden erfasst. Ergebnisse: Die Prävalenz von Schlaganfall und TIA war 7\%. Die Prävalenzraten für koronare Herzerkrankung und arterielle Hypertonie waren höher (14\% bzw. 53\%). Die 13 OSA-Patienten mit Schlaganfall waren ältere, übergewichtige Männer (Alter: $64 \pm 1,7$ Jahre, BMI : $31,4 \pm 1,1 \mathrm{~kg} / \mathrm{m}^{2}$ ). Sie litten unter einer mittel- bis höhergradig ausgeprägten OSA (AHI: $43 \pm 5 / \mathrm{h}$ ) und hatten fast alle einen Hypertonus. In allen Fällen lag ein ischämischer Schlaganfall vor, persistierende neurologische Ausfälle waren bei keinem Patienten zu beobachten. Die mittlere Latenzzeit zwischen dem Auftreten von Schlaganfall oder TIA und der Diagnose OSA war 41,9 $\pm 11,3$ Monate. Schlussfolgerungen: Zusammenfassend war die Prävalenz von Schlaganfall und TIA in unserer Untersuchung von insgesamt 187 OSA-Patienten $7 \%$. Aufgrund von „confounding factors“ wie Alter, Körpergewicht und Hypertonus sind prospektiv angelegte Fall-KontrollStudien notwendig, um das Risikopotenzial der OSA für die Entwicklung zerebrovaskulärer Erkrankungen definitiv beurteilen zu können.
\end{abstract}

Prevalence of Stroke and Transient Ischemic Attack (TIA) in Obstructive Sleep Apnea - a Retrospective Analysis of 187 Consecutive Patients: Background: Obstructive sleep apnea (OSA) is presumed to be significantly associated with cerebrovascular disease; however, there are no consistent epidemiological data providing proof of such a link. Hence, the aim of the present study was to determine the prevalence of stroke and transient ischemic attack (TIA) in patients with OSA. Patients and methods: 187 consecutive patients with a polysomnographically verified diagnosis of OSA who had been admitted to our sleep laboratory during 1/98-3/00 were retrospectively analysed. Results: The prevalence of stroke and TIA was $7 \%$. This rate was lower if compared with those for coronary artery

Pneumologie 2000; 54: 575-579

(c) Georg Thieme Verlag Stuttgart · New York ISSN 0934-8387
R. Schulz, H. Olschewski, F. Grimminger, W. Seeger

Medizinische Klinik II, Justus-Liebig-Universität, Gießen

(Direktor: Prof. Dr. med. W. Seeger) disease and arterial hypertension in the same study cohort ( $14 \%$ and $53 \%$ respectively). The 13 OSA patients with stroke were elderly overweight men (age: $64 \pm 1.7$ years, BMI: $31.4 \pm 1.1 \mathrm{~kg} / \mathrm{m}^{2}$ ). They had quite severe OSA (AHI: $43 \pm 5 / \mathrm{h}$ ) and almost all of them suffered from arterial hypertension. In all cases, the insult was caused by cerebral or brain stem ischemia. None of the patients presented with persistent neurological deficits. The mean latency period between the occurrence of stroke or TIA and the diagnosis of OSA was $41.9 \pm 11.3$ months. Conclusion: In summary, the prevalence of stroke or TIA in our series of 187 consecutive OSA patients was $7 \%$. Further prospective studies controlling for confounding factors such as age, body weight and arterial hypertension are needed to clarify the risk potential of OSA for the development of cerebrovascular disorders.

\section{Einleitung}

Die obstruktive Schlaf-Apnoe (OSA) ist wahrscheinlich kausal mit der systemarteriellen Hypertonie und der koronaren Herzerkrankung assoziiert [1,2]. Weniger eindeutig ist bisher die Beziehung zwischen der OSA und zerebrovaskulären Erkrankungen. Einige Autoren haben vorgeschlagen, dass die OSA einen unabhängigen Risikofaktor für die Entwicklung des Schlaganfalls darstellt, allerdings wurde in diesen Studien Schnarchen mit dem Vorhandensein einer OSA gleichgesetzt, ohne dass Polysomnographien durchgeführt wurden [3-7]. Neuere Untersuchungen haben zudem das Risikopotenzial des Schnarchens für das Auftreten von Schlaganfällen und transitorischen ischämischen Attacken (TIA) in Frage gestellt, nachdem die Odds Ratios für „confounding factors“ wie z.B. Alter, Geschlecht und Körpergewicht korrigiert worden waren [8-10].

Epidemiologische Studien haben eine hohe Prävalenz schlafbezogener Atemstörungen bei Patienten mit akutem Schlaganfall gezeigt. Zusammenfassend beträgt die Prävalenzrate der Schlaf-Apnoe in diesen Studien ca. 60-70\% [11-14]. Vergleichbare Untersuchungen zur Prävalenz von Schlaganfall und TIA bei Patienten mit gesicherter OSA liegen bisher nicht vor bzw. sind nicht unter dieser speziellen Fragestellung durchgeführt worden. Deshalb war das Ziel der vorliegenden

${ }^{1}$ Nach einem Vortrag gehalten auf der Tagung der Sektion „Nächtliche Atmungs- und Kreislaufstörungen“ der DGP und der Arbeitsgruppe Apnoe der DGSM am 12.11. 1999 in Hagen. 
Studie, über eine retrospektive Analyse einer größeren Gruppe von Patienten mit OSA Anhaltspunkte für diese Prävalenzraten zu erhalten.

\section{Patienten und Methodik}

\section{Patienten}

Alle Patienten, die im Zeitraum von 1/1998 bis 3/2000 wegen des Verdachts auf OSA in unserem Schlaflabor aufgenommen worden waren, wurden berücksichtigt. Vor der stationären Aufnahme war eine ambulante Screening-Untersuchung mit einem portablen Gerät erfolgt (Merlin, Heinen \& Löwenstein, Bad Ems). Weiterhin war ein von unserer Klinik entworfener, standardisierter Schlaf-Fragebogen ausgefüllt worden. Patienten, bei denen aufgrund der Screening-Untersuchung eine leichtgradige OSA vorlag, die alleine mit basistherapeutischen Maßnahmen wie z.B. Gewichtsabnahme behandelbar erschien, wurden von der Studie ausgeschlossen. Bei allen anderen Patienten wurde schließlich die Diagnose OSA durch eine Polysomnographie bestätigt (SIDAS GS, IfM GmbH, Wettenberg). Eine OSA wurde angenommen, falls die Patienten unter schlafbezogenen Symptomen litten (Schnarchen, beobachtete nächtliche Atempausen, exzessive Tagesschläfrigkeit) und der Apnoe-Hypopnoe-Index (AHI) > 10 pro Stunde totaler Schlafzeit (TST) war.

\section{Falldefinitionen}

Die Prävalenzraten für Schlaganfall und TIA und diejenigen für koronare Herzerkrankung und arteriellen Hypertonus wurden retrospektiv ermittelt. Schlaganfall oder TIA wurde definiert als das plötzliche Auftreten von neurologischen Defiziten, z.B. in Form von sensomotorischen Symptomen, Sehstörungen oder einer Aphasie.

Bei der TIA mussten sich die Symptome innerhalb von 24 Stunden vollständig zurückgebildet haben, ansonsten wurde von einem Apoplex ausgegangen. Entsprechende Fälle wurden anhand von Anamnese, körperlicher Untersuchung und den Ergebnissen bildgebender Verfahren (Computertomographie oder Kernspintomographie des Schädels) erfasst. Bei den betroffenen Patienten wurden die Schlaganfälle näher charakterisiert: Art und Lokalisation des Schlaganfalles (ischämisch oder hämorrhagisch, lokalisiert im Hirnstamm oder im Großhirn), Persistenz neurologischer Defizite sowie Latenzperiode zwischen dem Auftreten des Schlaganfalles und der Diagnose OSA.

Eine koronare Herzerkrankung (KHK) wurde diagnostiziert, wenn eine typische klinische Symptomatik bestand (z.B. belastungsabhängige Angina pectoris), Infarktzeichen in Elektro- oder Echokardiographie vorlagen oder frühere Koronarangiographien signifikante Stenosen gezeigt hatten $(>50 \%$ des Gefäßdurchmessers). Ein arterieller Hypertonus wurde angenommen bei Blutdruckwerten, die mehrfach 140/ $90 \mathrm{mmHg}$ überschritten oder bei bekanntem und medikamentös eingestellten Hypertonus.

Eine chronischobstruktive Atemwegserkrankung (COPD) wurde definiert als eine obstruktive Ventilationsstörung $\left(\mathrm{FEV}_{1}\right.$ $<80 \%$ vom Soll) in Verbindung mit produktivem Husten über mindestens 3 Monate während 2 aufeinander folgender Jahre.
Asthma bronchiale wurde angenommen, falls es sich um anfallsweise aufgetretene, reversible bronchiale Obstruktionen handelte. Schließlich wurden die Rauchgewohnheiten der Patienten erfasst.

\section{Statistik}

Die Daten sind als Absolutzahlen und als Prozent bezogen auf die Gesamtzahl der Patienten oder als Mittelwert \pm SEM angegeben. Die Charakteristika der OSA-Patienten mit/ohne Apoplex und TIA wurden miteinander mit Hilfe des Student-tTest für ungepaarte Daten verglichen. Ein p-Wert $<0,05$ wurde als signifikant angesehen.

\section{Ergebnisse}

\section{Charakteristika der Gesamtpopulation}

Bei insgesamt 187 Patienten wurde im angegebenen Zeitraum die Diagnose einer OSA gestellt. Die Patientencharakteristika sind in Tab. 1 zusammengefasst. Das mittlere Alter entsprach der von der OSA am häufigsten betroffenen Lebensdekade (50-60 Jahre). Das männliche Geschlecht war in unserem Kollektiv überproportional häufig vertreten (Geschlechtsverhältnis Männer/Frauen ca. 8:1). Die Patienten litten unter einer mittel- bis höhergradigen OSA.

Bei 4/5 der Patienten wurde eine nasale CPAP- oder BiPAPTherapie eingeleitet. Bei den restlichen Patienten bestand in der Mehrzahl der Fälle nur eine leichtgradige OSA ohne CPAP/ BiPAP-Indikation, sodass eine alleinige Gewichtsreduktion

Tab. 1 Charakteristika aller OSA-Patienten $(n=187)$

\begin{tabular}{|c|c|}
\hline Charakteristika aller OSA-Patienten & $(n=187)$ \\
\hline Alter - Jahre & $55 \pm 0,8$ \\
\hline Geschlecht - männlich/weiblich & $167 / 20$ \\
\hline $\mathrm{BMI}-\mathrm{kg} / \mathrm{m}^{2}$ & $31,6 \pm 0,4$ \\
\hline $\mathrm{AHI}-\mathrm{n} / \mathrm{h}$ & $45 \pm 2$ \\
\hline mittlere Apnoelänge (s) & $20 \pm 1$ \\
\hline $\mathrm{SaO}_{2}<90 \%-\%$ der TST & $23,3 \pm 1,6$ \\
\hline minimaler $\mathrm{SaO}_{2}$-Wert (\%) & $68,6 \pm 0,8$ \\
\hline $\mathrm{paO}_{2}$ im Wachzustand $(\mathrm{mmHg})$ & $73,4 \pm 0,7$ \\
\hline CPAP - n (\%) & $134(72)$ \\
\hline CPAP Druck - $\mathrm{cm} \mathrm{H}_{2} \mathrm{O}$ & $9,2 \pm 0,2$ \\
\hline BiPAP - n (\%) & $15(8)$ \\
\hline Schlaganfall/TIA - n (\%) & $13(7)$ \\
\hline Arterielle Hypertonie - n (\%) & $99(53)$ \\
\hline Koronare Herzerkrankung - n (\%) & $26(14)$ \\
\hline Diabetes mellitus - n (\%) & $22(12)$ \\
\hline Hyperlipidämie - n (\%) & $86(46)$ \\
\hline Rauchen - n (\%) & $28(15)$ \\
\hline COPD/Asthma - n (\%) & $30(16)$ \\
\hline
\end{tabular}

Die Daten sind als Absolutzahlen und als Prozent bezogen auf die Gesamtzahl der Patienten oder als Mittelwert \pm SEM angegeben. BMI = Body Mass Index, AHI = Apnoe-Hypopnoe-Index; $\mathrm{SaO}_{2}=$ Sauerstoffsättigung, TST - totale Schlafzeit; $\mathrm{paO}_{2}$ arterieller Sauerstoffpartialdruck; COPD=chronisch-obstruktive Atemwegserkrankung 
empfohlen wurde. 8 Patienten lehnten eine CPAP/BiPAPTherapie schon initial $a b$, bei 2 Patienten wurde primär eine Operation durchgeführt (1-mal Tonsillektomie, 1-mal kieferchirurgische OP).

13 der 187 Patienten hatten einen Schlaganfall oder eine TIA erlitten (7\%), 26 Patienten hatten eine koronare Herzerkrankung (14\%) und 99 Patienten einen arteriellen Hypertonus (53\%). 30 Patienten litten unter COPD oder einem Asthma bronchiale (16\%) und 28 Patienten waren Raucher (15\%).

\section{Charakteristika der OSA-Patienten mit Schlaganfall/TIA}

Die Charakteristika der 13 Patienten mit Schlaganfall und TIA sind in der Tab. 2 denjenigen der Patienten ohne zerebrovaskuläre Erkrankungen gegenübergestellt. Alle Patienten mit Schlaganfall/TIA waren männlichen Geschlechts. Im Mittel waren sie 9 Jahre älter als die Patienten ohne Apoplex, der mittlere BMI war vergleichbar. Die Anzahl der Apnoen und Hypopnoen und die mittlere Apnoelänge waren ähnlich wie bei den Patienten ohne zerebrovaskuläre Ereignisse, allerdings wiesen die Schlaganfallpatienten einen Trend zu deutlicheren nächtlichen Sauerstoffentsättigungen auf. Dies war darauf zurückzuführen, dass in dieser Gruppe wahrscheinlich altersbedingt niedrigere Sauerstoffpartialdrucke im Wachzustand vorlagen.

Weiterhin zeigten die OSA-Patienten mit Apoplex/TIA eine ausgeprägtere kardiovaskuläre Komorbidität als die OSAPatienten ohne Schlaganfall und TIA. Fast alle dieser Patienten litten unter einer arteriellen Hypertonie und eine KHK war ca. 3-mal häufiger anzutreffen als bei den Patienten ohne zerebrovaskuläre Erkrankungen. Ein Diabetes mellitus war ebenfalls häufiger bei den Schlaganfallpatienten vorhanden, während sich die Häufigkeit von Fettstoffwechselstörungen zwischen beiden Gruppen nicht signifikant unterschied.

\section{Schlaganfall-Charakteristika}

9 Patienten hatten einen Schlaganfall erlitten, 4 Patienten eine TIA. Bei den Schlaganfällen handelte es sich ausnahmslos um ischämische Insulte, während intrazerebrale Blutungen in keinem Fall beobachtet wurden.

Die Schlaganfälle waren gleichmäßig zwischen Hirnstammarealen und beiden Großhirnhemisphären verteilt. Bei keinem Patienten wurden zum Zeitpunkt der Polysomnographie persistierende neurologische Defizite festgestellt. Die mittlere Latenzzeit zwischen Schlaganfall oder TIA und der Diagnosestellung OSA war ungefähr $3 \frac{1}{2}$ Jahre. Nur bei 2 Patienten hatte sich der Apoplex unmittelbar vor der Aufnahme im Schlaflabor ereignet.

\section{Diskussion}

Wir fanden eine Prävalenzrate von Schlaganfall und TIA bei OSA von 7\%. Zwei frühere Studien, die weniger Patienten umfassten und sich nicht primär mit der Prävalenz zerebrovaskulärer Erkrankungen bei OSA beschäftigten, haben ähnliche bzw. etwas höhere Zahlen erbracht. Olson et al. [8] ermittelten eine vergleichbare Prävalenzrate (6 von 79 Patienten bzw. 8\%), während Pendlebury et al. [15] in ihrem Patientengut eine höhere Prävalenz von $18 \%$ fanden (10 von
55 Patienten). Die Häufigkeiten von koronarer Herzerkrankung und arterieller Hypertonie in unserer Untersuchung waren größer als diejenigen von Apoplex und TIA und stehen in Übereinstimmung mit Daten aus der Literatur $[8,16,17]$.

Verschiedene Einflussgrößen können dazu beigetragen haben, dass die Prävalenz von Schlaganfall und TIA bei OSA in unserer Studie sowohl unter- als auch überschätzt wurde. Da die OSA-Patienten mit Schlaganfall zum Zeitpunkt der Polysomnographie keine neurologischen Residuen aufwiesen, ist es denkbar, dass Patienten mit bleibenden Ausfällen und Verdacht auf OSA nicht weiter abgeklärt werden und somit unerkannt bleiben. Weiterhin ist es möglich, dass durch den retrospektiven Charakter der vorliegenden Untersuchung Informationen verloren gingen und einige Patienten fälschlicherweise nicht als Apoplex-Patienten klassifiziert wurden. Auf der anderen Seite ist zu bedenken, dass vor der Aufnahme in das Schlaflabor eine Patientenselektion vorgenommen wurde, die zum Ausschluss von OSA-Patienten mit nur geringgradiger nächtlicher Atemstörung führte. Wären diese Patienten mit berücksichtigt worden, wäre die Prävalenz von Schlaganfall und TIA vermutlich geringer ausgefallen. Schließlich ist zu berücksichtigen, dass die OSA-Patienten unseres Schlaflabors wahrscheinlich multimorbider sind als solche, die an anderen Kliniken oder sogar ambulant behandelt werden. Dies könnte ebenfalls zu einer falsch $\mathrm{zu}$ hohen Prävalenz von Apoplex und TIA in der vorliegenden Untersuchung geführt haben.

Bemerkenswert ist die Differenz der Prävalenzraten von Schlaganfall bei OSA versus Schlaf-Apnoe bei Patienten mit Schlaganfall. Zum Teil ist dies wohl darauf zurückzuführen, dass die Prävalenz schlafbezogener Atemstörungen bei Schlaganfallpatienten bisher überschätzt worden ist. Beim Großteil der angeführten Studien wurde eine Polysomnographie in der Akutphase des Schlaganfalls, also während der ersten Wochen nach dem neurologischen Ereignis durchgeführt [11-14]. Es ist aber bekannt, dass nächtliche Atemstörungen in Folge eines Schlaganfalles sich mit der Rekonstitution neurologischer Funktionen wieder zurückbilden können [18].

Aufgrund unserer Studie ist es nicht möglich, das Risikopotenzial der OSA für die Entstehung von Schlaganfall oder TIA zu bestimmen. Dazu muss die Prävalenzrate dieser Erkrankungen bei OSA zunächst mit derjenigen in der Normalbevölkerung verglichen werden. Eine solche Kontrollgruppe wurde von uns nicht untersucht, es mag aber gerechtfertigt sein, einen Vergleich mit einem historischen Kollektiv vorzunehmen. Eine im Geltungsbereich der AOK Dortmund durchgeführte Erhebung an einer 5\%-Zufalls-Stichprobe von ca. 7000 Versicherten fand innerhalb der mit unseren OSAPatienten vergleichbaren Altersgruppe der 50-60-Jährigen eine Schlaganfall-Prävalenzrate von 1,1\% [19]. Dies würde bedeuten, dass die Prävalenz von Schlaganfall und TIA bei OSA ungefähr 6-mal größer ist als in der Allgemeinbevölkerung. Bevor aber festgestellt werden kann, dass die OSA per se zur Entstehung von zerebrovaskulären Erkrankungen führt, muss der Einfluss von sogen. „confounding factors“ wie Alter, Körpergewicht und Blutdruck berücksichtigt werden. Diese stellen unabhängige Schlaganfall-Risikofaktoren dar [20] und lagen auch bei unseren OSA-Patienten mit Schlaganfall vor. 


\begin{tabular}{llll}
\hline Schlaganfall/TIA & ja & nein & p-Wert \\
\hline n (\%) & $13(7)$ & $174(93)$ & \\
Alter - Jahre & $64 \pm 1,7$ & $55 \pm 0,8$ & $<0,01$ \\
Geschlecht - männlich/weiblich & $13 / 0$ & $154 / 20$ & $<0,01$ \\
BMI - kg/m & $31,4 \pm 1,1$ & $31,6 \pm 0,4$ & n.s. \\
AHI - n/h & $43 \pm 5$ & $45 \pm 2$ & n.s. \\
mittlere Apnoelänge (sec.) & $21 \pm 2$ & $20 \pm 1$ & n.s. \\
SaO $<90 \%$ \% der TST & $29,9 \pm 6,4$ & $22,7 \pm 1,6$ & $=0,07$ \\
minimaler SaO -Wert (\%) & $67,8 \pm 2,0$ & $68,7 \pm 0,9$ & n.s. \\
paO 2 im Wachzustand (mmHg) & $67,3 \pm 2,6$ & $73,8 \pm 0,7$ & $<0,01$ \\
Arterielle Hypertonie - n (\%) & $11(85)$ & $88(51)$ & $<0,01$ \\
Koronare Herzerkrankung - n (\%) & $5(38)$ & $21(12)$ & $<0,01$ \\
Diabetes mellitus - n (\%) & $4(31)$ & $18(10)$ & $<0,01$ \\
Hyperlipidämie - n (\%) & $7(54)$ & $79(45)$ & n.s. \\
Rauchen - n (\%) & $2(15)$ & $26(15)$ & n.s. \\
COPD/Asthma - n (\%) & $2(15)$ & $28(16)$ & n.s. \\
TIA - n (\%) & $4(31)$ & - & \\
Schlaganfall - (\%) & $9(69)$ & - & \\
- Hemisphäre & 5 & & \\
- Hirnstamm & 4 & & \\
Latenzzeit Schlaganfall/TIA - Diagnose OSA & $41,9 \pm 11,3$ & & \\
(Monate) & & & \\
\hline
\end{tabular}

Tab. 2 Charakteristika der OSA-Patienten mit/ohne Schlaganfall und TIA

Die Daten sind als Absolutzahlen und als Prozent bezogen auf die Gesamtzahl der Patienten oder als Mittelwert \pm SEM angegeben. BMI = Body Mass Index, AHI - Apnoe-Hypopnoe-Index; $\mathrm{SaO}_{2}=$ Sauerstoffsättigung, TST - totale Schlafzeit; $\mathrm{paO}_{2}$ arterieller Sauerstoffpartialdruck; $\mathrm{COPD}=$ chronisch-obstruktive Atemwegserkrankung, n.s. = nicht signifikant

Deshalb ist es notwendig, Fall-Kontroll-Studien durchzuführen, die Patienten ohne OSA aber mit einem ähnlichen Spektrum an Begleiterkrankungen mit einbeziehen. Weiterhin wäre ein prospektiver Charakter einer solchen Untersuchung zu fordern, um Index-Patienten möglichst vollständig und zuverlässig zu erfassen.

Abschließend soll erwähnt werden, dass einige neuere Beobachtungen auf die Möglichkeit hindeuten, dass die OSA tatsächlich als Risikofaktor für den Schlaganfall anzusehen ist. Erstens haben Doppler-Messungen gezeigt, dass es bei OSA zu zyklischen Veränderungen der zerebralen Blutflussgeschwindigkeit kommt [21]. Diese Veränderungen sind wahrscheinlich Folge apnoe-assoziierter Blutdruck- und Sympathikusaktivierungen und führen $\mathrm{zu}$ vermehrtem „shear stress" auf die Gefäßwand. Zweitens konnte nachgewiesen werden, dass die zerebrale Vasodilatation bei OSA reduziert ist und durch eine effektive CPAP-Therapie wieder normalisiert werden kann [22,23]. Grundlage hierfür dürfte eine verminderte endotheliale Bildung von Stickstoffmonoxid sein [24]. Drittens wurde anhand der Analyse von lateralen Cephalometrie-Bildern demonstriert, dass OSA-Patienten häufiger verkalkte Carotiden haben als gesunde Kontrollpersonen $[25,26]$. Es könnte also bei der OSA durch Störungen der vaskulären Homöostase zu einer akkzelerierten Atherosklerose der extra- (und intra-)kraniellen Gefäße mit der erhöhten Gefahr des Schlaganfalls kommen. Schließlich ist zu bedenken, dass die bereits erwähnte erhöhte Sympathikusaktivität bei OSA auch im Wachzustand nachweisbar ist $[27,28]$ und über die Entwicklung eines arteriellen Hyper- tonus indirekt zu einem erhöhten Schlaganfallrisiko beitragen könnte.

Zusammenfassend betrug in unserer retrospektiven Analyse von insgesamt 187 konsekutiven Patienten mit polysomnographisch dokumentierter OSA die Prävalenz von Schlaganfall und TIA 7\%. Aufgrund von „confounding factors“ wie Alter, Körpergewicht und Hypertonus sind prospektiv angelegte Fall-Kontroll-Studien notwendig, um das Risikopotenzial der OSA für die Entwicklung zerebrovaskulärer Erkrankungen definitiv beurteilen zu können.

\section{Literatur}

${ }^{1}$ Young T, Peppard P, Palta M, Hla M, Finn L, Morgan B, Skatrud J. Population-based study of sleep-disordered breathing as a risk factor for hypertension. Arch Intern Med 1997; 157: 1746- 1752

${ }^{2}$ Peker Y, Kraiczi H, Hedner J, Löth S, Johansson A, Bende M. An independent association between obstructive sleep apnoea and coronary artery disease. Eur Respir J 1999; 14: 179-184

${ }^{3}$ Koskenvuo M, Kaprio J, Tekakivi T, Partinen H, Heikkila K, Sarna S. Snoring as a risk factor for ischemic heart disease and stroke in men. Br Med J 1987; 294: 16 - 19

${ }^{4}$ Palomaki H, Partinen M, Juvela S, Kaste M. Snoring as a risk factor for sleep-related brain infarction. Stroke 1989; 20: 1311 1315

${ }^{5}$ Palomaki H. Snoring and the risk of ischemic brain infarction. Stroke 1991; 22: 1021 - 1025

${ }^{6}$ Spriggs DA, French JM, Murdy JM, Curless RH, Bates D, James OF. Snoring increases the risk of stroke and adversely affects prognosis. QJ Med 1992; 83: 555-562 
${ }^{7}$ Neau JP, Meurice JC, Paquereau J, Chavagnat JJ, Ingrand P, Gil R. Habitual snoring as a risk factor for brain infarction. Acta Neurol Scand 1995; 92: 63-68

${ }^{8}$ Olson G, King MT, Hensley MJ, Saunders NA. A community study of snoring and sleep-disordered breathing: health outcomes. Am J Respir Crit Care Med 1995; 152: 717 - 720

${ }^{9}$ Davies PD, Oates J, Rodgers H, James OFW, Gibson GJ. Objective comparison of snoring in stroke survivors and controls. Am J Respir Crit Care Med 1997; 155 (Suppl.): A843

${ }^{10}$ Davies PD, Oates J, Rodgers H, James OFW, Gibson GJ. Snoring and stroke: a case-control study in first-ever stroke. Am J Respir Crit Care Med 1997; 155 (Suppl.): A846

${ }^{11}$ Bassetti C, Aldrich MS, Chervin RD, Quint D. Sleep apnea in patients with transient ischemic attack and stroke: a prospective study of 59 patients. Neurology 1996; 47: 1167-1173

${ }^{12}$ Bassetti C, Aldrich MS, Quint D. Sleep-disordered breathing in patients with acute supra- and infratentorial strokes. A prospective study of 39 patients. Stroke 1997; 28: 1765-1772

${ }^{13}$ Bassetti C, Aldrich MS. Sleep apnea in acute cerebrovascular diseases: final report on 128 patients. Sleep 1999; 22: 217-223

${ }^{14}$ Wessendorf TE, Teschler H, Wang YM, Konietzko N, Thilmann AF. Sleep-disordered breathing among patients with first-ever stroke. J Neurol 2000; 247: 41 - 47

${ }^{15}$ Pendlebury ST, Pepin JL, Levy P. Natural evoluation of moderate sleep apnoea syndrome: significant progression over a mean of 17 months. Thorax 1997; 52: $872-878$

${ }^{16}$ Carlson J, Hedner J, Ejnell H, Peterson L. High prevalence of hypertension in sleep apnea patients independent of obesity. Am J Respir Crit Care Med 1994; 150: 72 - 77

${ }^{17}$ Maekawa M, Shiomi T, Usui K, Sasanabe R, Kobayashi T. Prevalence of ischemic heart disease among patients with sleep apnea syndrome. Psychiatry Clin Neurosci 1998; 52: 219-220

18 Parra O, Arboix A, Bechich S, Garcia-Eroles L, Montserrat JM, Lopez JA, Ballester E, Guerra JM, Sopena JJ. Time course of sleeprelated breathing disorders in first-ever stroke or transient ischemic attack. Am J Respir Crit Care Med 2000; 161: 375 - 380

${ }^{19}$ Hartlapp V, Koster I, von Ferber L. Häufigkeit und Verteilung von Apoplexie und transitorischer ischämischer Attacke: Sekundäranalyse von Verwaltungsdaten von Versicherten der AOK Dortmund. Soz Präventivmed 1996; 41: 178 - 184

${ }^{20}$ Stegmayr B, Asplund K, Kuulasmaa K, Rajakangas AM, Thorvaldsen P, Tuomilehto J. Stroke incidence and mortality correlated to stroke risk factors in the WHO MONICA Project. An ecological study of 18 populations. Stroke 1997; 28: 1367-1374

${ }^{21}$ Balfors EM, Franklin KA. Impairment of cerebral perfusion during obstructive sleep apneas. Am J Respir Crit Care Med 1994; 150: 1587-1591

${ }^{22}$ Placidi F, Diomedi M, Cupini LM, Bernardi G, Silvestrini M. Impairment of daytime cerebrovascular reactivity in patients with obstructive sleep apnoea syndrome. J Sleep Res 1998; 7: 288-292

${ }^{23}$ Diomedi M, Placidi F, Cupini LM, Bernardi G, Silvestrini M. Cerebral hemodynamic changes in sleep apnea syndrome and effect of continuous positive airway pressure treatment. Neurology 1998; 51: $1051-1056$

${ }^{24}$ Schulz R, Schmidt D, Blum A, Lopes-Ribeiro X, Lücke C, Mayer K, Olschewski H, Seeger W, Grimminger F. Decreased plasma levels of nitric oxide derivatives in obstructive sleep apnoea - response to CPAP therapy. Thorax, im Druck.

${ }^{25}$ Friedlander AH, Yueh R, Littner MR. The prevalence of calcified carotid artery atheromas in patients with obstructive sleep apnea syndrome. J Oral Maxillofac Surg 1998; 56: 950-954

${ }^{26}$ Friedlander AH, Friedlander IK, Yueh R, Littner MR. The prevalence of carotid atheromas seen on panoramic radiographs of patients with obstructive sleep apnea and their relation to risk factors for atherosclerosis. J Oral Maxillofac Surg 1999; 57: 516 521

${ }^{27}$ Carlson JT, Hedner J, Elam M, Ejnell H, Sellgren J, Wallin BG. Augmented resting sympathetic nerve activity in awake patients with obstructive sleep apnea. Chest 1993; 103: 1763-1768

${ }^{28}$ Waradekar NV, Sinoway LI, Zwillich CW, Leuenberger UA. Influence of treatment on muscle sympathetic nerve activity in sleep apnea. Am J Respir Crit Care Med 1996; 153: 1333-1338

\section{Dr. med. R. Schulz}

Medizinische Klinik II Justus-Liebig-Universität Klinikstr. 36 35392 Gießen 